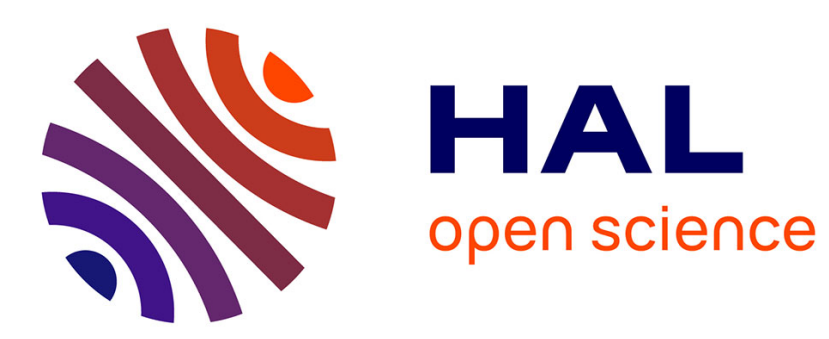

\title{
Are non-sexual models appropriate for predicting the impact of virus-vectored immunocontraception?
}

Anne Deredec, Luděk Berec, David S. Boukal, Franck Courchamp

\section{To cite this version:}

Anne Deredec, Luděk Berec, David S. Boukal, Franck Courchamp. Are non-sexual models appropriate for predicting the impact of virus-vectored immunocontraception?. Journal of Theoretical Biology, 2008, 250 (2), pp.281-290. 10.1016/j.jtbi.2007.09.037 . hal-02665551

\section{HAL Id: hal-02665551 https://hal.inrae.fr/hal-02665551}

Submitted on 31 May 2020

HAL is a multi-disciplinary open access archive for the deposit and dissemination of scientific research documents, whether they are published or not. The documents may come from teaching and research institutions in France or abroad, or from public or private research centers.
L'archive ouverte pluridisciplinaire HAL, est destinée au dépôt et à la diffusion de documents scientifiques de niveau recherche, publiés ou non, émanant des établissements d'enseignement et de recherche français ou étrangers, des laboratoires publics ou privés. 


\title{
Are non-sexual models appropriate for predicting the impact of virus-vectored immunocontraception?
}

\author{
Anne Deredec $^{\mathrm{a}, 1}$, Luděk Berec ${ }^{\mathrm{b}, *}$, David S. Boukal ${ }^{\mathrm{b}, \mathrm{c}, \mathrm{d}}$, Franck Courchamp ${ }^{\mathrm{a}}$ \\ ${ }^{a}$ Ecologie, Systématique et Evolution, Université Paris-Sud XI, Bâtiment 362, 91405 Orsay Cedex, France \\ ${ }^{\mathrm{b}}$ Department of Theoretical Ecology, Institute of Entomology, Biology Centre ASCR, Branišovská 31, 37005 České Budějovice, Czech Republic \\ ${ }^{\mathrm{c}}$ Institute of Marine Research, P.O. Box 1870 Nordnes, N5817 Bergen, Norway \\ ${ }^{\mathrm{d}}$ Department of Biology, University of Bergen, P.O. Box 7800, N5020 Bergen, Norway
}

Received 14 March 2007; received in revised form 18 September 2007; accepted 26 September 2007

Available online 29 September 2007

\begin{abstract}
In response to the need to efficiently control mammal pest populations while avoiding unnecessary suffering, applied and theoretical ecologists have recently focused on virus-vectored immunocontraception (VVIC). So far, modellers have only considered a non-sexual approach (models of sexually reproducing populations without explicitly discerning between the sexes), which appears dubious in view of the sex-specificity of VVIC agents. In this paper, we derive and compare predictions of non-sexual and two-sex models of the spread of a VVIC agent in a host population in order to assess the adequacy of non-sexual models in this context. Our results show that predictions of non-sexual and two-sex models generally diverge and that non-sexual models often fail to predict the control impact of VVIC. We thus recommend using two-sex models, especially if the mating system and life history of the target species are known. Our analysis also shows that female-specific viruses generally give better results than male-specific ones, and suggests that virus choice should focus more on its sterilizing power rather than transmission efficiency.
\end{abstract}

(C) 2007 Elsevier Ltd. All rights reserved.

Keywords: Biological control; Mammal pest; Population dynamics; Sterilization; Two-sex model

\section{Introduction}

Biological invasions are becoming a major concern for ecologists and biodiversity managers alike (Vitousek et al., 1997). Among invasive species, introduced mammals have reportedly caused more problems than any other vertebrate group (Ebenhard, 1988; Lever, 1994). Probably in part due to the general lack of naturally occurring terrestrial mammals on most remote islands (Atkinson, 2001), mammals are also responsible for the best documented and most spectacular ecological disturbances resulting from biological invasions (Courchamp et al., 2003).

\footnotetext{
*Corresponding author. Tel.: + 420387772327 ; fax: +420385310354.

E-mail addresses: a.deredec@imperial.ac.uk (A. Deredec), berec@entu.cas.cz (L. Berec), boukal@entu.cas.cz (D.S. Boukal), franck.courchamp@u-psud.fr (F. Courchamp).

${ }^{1}$ Present address: NERC Centre for Population Biology, Imperial College London, Silwood Park Campus, Ascot, Berkshire, SL5 7PY, UK.
}

Various control programs are being increasingly used in an attempt to limit and, whenever possible, reverse biodiversity erosion. In particular, 153 mammal eradication programs were completed at the end of 2001 on New Zealand offshore islands alone (C.R. Veitch, personal communication). Control programs are also important on mainland, although the areas involved often preclude complete eradication with current methods and limit control efforts to alleviation.

Logistical and economical problems still limit efficiency of mammal control. In particular, areas, which are large, difficult to access, or have a low pest density are extremely difficult if not impossible to cover by hunters, traps or poisonous baits. As dissemination of control agents constitutes a major limitation in many control programs, research has recently focused on biological control. Biological control relies upon self-disseminating natural enemies such as viruses which may have the double advantage of economical viability and high control success. 
However, use of most pathogens is unethical as they inflict unnecessary suffering before killing the host.

Recently, research effort has turned towards virusvectored immunocontraception (VVIC), a new form of biological control that retains the advantages of selfdissemination of control agents while avoiding the unethical aspects of animal suffering. VVIC is based on a sterilization process that induces the immune system of an individual to attack its own reproductive cells-infecting an individual with a protein derived from the follicular layers activates production of antibodies against its own gametes, thereby blocking fertilization (Bradley et al., 1997; Tyndale-Biscoe, 1994). VVIC agents are viruses which are genetically modified to carry a gene encoding the reproductive protein of a target species (Tyndale-Biscoe, 1994). The use of modified, species-specific viruses thus allows dissemination of a control agent through a pest population regardless of its area of distribution, accessibility and density, and combines the advantages of high specificity and optimal dissemination. This potentially powerful new technique appears most appropriate for rodents and small herbivores, such as rabbits and possums (Cowan, 1996; McLeod and Twigg 2006; Rodger, 1997; Smith et al., 1997), yet it could also be very efficient for control of small carnivores such as cats and foxes (Bradley et al., 1997; Courchamp and Cornell, 2000; Pech et al., 1997; Verdier et al., 1999).

Before VVIC is accepted as a plausible technique of controlling mammal pests, its efficiency (and safety) must be reliably approved. A modified virus must be capable of (1) generating a sterilizing immune response after natural infection in the target species (while being harmless to others) (Holland and Jackson, 1994; Holland et al., 1997) and (2) spreading and persisting within the population and stimulating a marked decrease in host numbers. Although it is not possible to adequately evaluate population-level efficiency of VVIC without an experimental field release, there is a strong demand among applied ecologists for theoretical studies that would provide a preliminary assessment of the technique and guide such experiments (Barlow, 2000).

To this end, several host-parasite models describing the spread of sterilizing pathogens within host populations have already been developed. Some of them have been partially tailored to brushtail possums Trichosurus vulpecula (Barlow, 1994), domestic cats Felis catus (Courchamp and Cornell, 2000), and European rabbits Oryctolagus cuniculus (Hood et al., 2000; McLeod and Twigg, 2006). Others have been more general and focused on diverse evolutionary issues in simple, host-sterilizing-pathogen systems (O'Keefe and Antonovics, 2002; Thrall and Antonovics, 1997; Thrall et al., 1998).

Except for a short paragraph in Barlow (1994) and a bioeconomic analysis carried out by McLeod and Twigg (2006), studies modelling the impact of VVIC neither considered sex-specificity of the virus nor explored the extent to which predictions of two-sex models (i.e. models of sexually reproducing populations that explicitly discern between the sexes) and non-sexual models (i.e. models of sexually reproducing populations that do not discern between the sexes) may deviate (Barlow, 1997; Courchamp and Cornell, 2000; Hood et al., 2000).

We believe that non-sexual models may not provide accurate predictions for at least three reasons. First, VVIC agents are sex-specific. While the virus is infecting both sexes, it affects only one of them: a male-specific virus will render sterile only males but both males and females will be carrying and spreading it. Male- and female-specific viruses may thus affect target populations differently (Barlow, 1994; Bomford, 1990). Second, except for sexually transmitted diseases (STD), both non-mating and mating encounters promote the spread of the virus. Patterns of encounters between individuals and hence of virus transmissions may differ in the mating and non-mating phases of the reproductive cycle, and be shaped by diverse mating systems (Sarre et al., 2000; Thrall et al., 2000). Last but not least, distinction between males and females has been shown to alter predictions of analogous non-sexual models in other contexts (Boukal and Berec, 2002; Legendre et al., 1999; Lindström and Kokko, 1998; Rankin and Kokko, 2007).

In this paper, we develop and compare predictions of a two-sex model of a host population controlled by an immunocontraceptive virus and of its non-sexual counterpart to assess whether VVIC models should explicitly account for males and females.

\section{Methods}

\subsection{Modelling framework}

Dynamics of sexually reproducing populations have often been described by non-sexual models (as defined above, models of sexually reproducing populations that do not discern between the sexes) because of their relative simplicity and analytical tractability. Extending such models to account for both sexes is not straightforward and no standard recipe is currently available. Here, we develop a stochastic, individual-based model which follows the life history of each individual, and proceeds in discrete time steps representing reproductive cycles. This modelling approach goes beyond the mainstream of current VVIC studies; most of them are modified epidemiology models based on ordinary differential equations. We know of only one study that develops its own stochastic, individualbased model: Arthur et al. (2007) use it to examine how competition between a wild type and an engineered form of a virus affects the efficiency of VVIC technique in controlling annual population outbreaks of the house mouse Mus musculus domesticus in south-eastern Australia.

We consider a promiscuous mating system, common to mammals that are the prime target for VVIC, and assume that infection is directly transmitted between animals by sufficiently close contacts. We develop both a two-sex 
model and a corresponding non-sexual variant, and use the subscripts $s$ and $a$, respectively, to distinguish them formally. Development of our own non-sexual model is essential for proper comparisons: it is much easier to derive a properly corresponding non-sexual model from a two-sex model than the other way round. Nonetheless, our nonsexual model has all the 'standard' ingredients of mainstream non-sexual models. For the sake of comparison, we also study the two types of virus (male and female specific) in the non-sexual model. Altogether, we thus explore four model variants (non-sexual and two-sex model, each with male- and female-specific virus). All symbols used in this study are summarized in Table 1.

The simulation protocol, common to all model variants, consists of a pre-control and control phase. In the precontrol phase, we introduce 200 infection-free individuals (100 males and 100 females in the two-sex model) and let the host population reach equilibrium, following its dynamics for 100 reproductive cycles. In the control phase, we initially inoculate 20 randomly chosen individuals and then regularly, every second reproductive season, infect additional four susceptible individuals (initially 10 individuals and then regularly two of each sex in the two-sex model). We follow such a population for 300 reproductive cycles to let it achieve new equilibrium. Regular inoculation prevents the occurrence of infection fadeouts, whereby all infected individuals would die out at a population bottleneck due to demographic stochasticity and the population now composed of only susceptible individuals would recover to its pre-control level. Test simulations

Table 1

List of symbols used in this study

\begin{tabular}{|c|c|}
\hline Symbol & Meaning \\
\hline$\beta$ & $\begin{array}{l}\text { Transmission efficiency (probability of virus transmission } \\
\text { upon a sufficiently close contact between a susceptible and an } \\
\text { infected individual) }\end{array}$ \\
\hline$\gamma$ & $\begin{array}{l}\text { Sterilizing power (probability that an infected individual of } \\
\text { the target species becomes sterile) }\end{array}$ \\
\hline$N$ & Adult population size \\
\hline$K$ & Carrying capacity of the environment \\
\hline$b_{a}$ & $\begin{array}{l}\text { Number of offspring produced by an individual in the non- } \\
\text { sexual model per reproductive cycle }\end{array}$ \\
\hline$\Lambda_{a}(N)$ & $\begin{array}{l}\text { Total number of (non-mating) encounters per reproductive } \\
\text { cycle in the non-sexual model }\end{array}$ \\
\hline$P$ & $\begin{array}{l}\text { Probability that an infected individual in the non-sexual } \\
\text { model gives rise to progeny }\end{array}$ \\
\hline$h$ & $\begin{array}{l}\text { Number of females every male mates with in a reproductive } \\
\text { cycle }\end{array}$ \\
\hline$b_{s}$ & $\begin{array}{l}\text { Number of offspring produced by a mated female per } \\
\text { reproductive cycle in the two-sex model }\left(=2 b_{a}\right)\end{array}$ \\
\hline$\Lambda_{s}(N)$ & $\begin{array}{l}\text { Total number of non-mating encounters per reproductive } \\
\text { cycle in the two-sex model }\end{array}$ \\
\hline$\lambda_{a}, \lambda_{s}$ & $\begin{array}{l}\text { Parameters scaling total numbers of non-mating encounters } \\
\Lambda_{a} \text { and } \Lambda_{s}\end{array}$ \\
\hline$E$ & $\begin{array}{l}\text { Maximum number of non-mating encounters per } \\
\text { reproductive cycle, that is, total number of non-mating } \\
\text { encounters at } N=K\end{array}$ \\
\hline
\end{tabular}

showed that regular inoculations are important for virus persistence in the host population but the results stay virtually insensitive to their magnitude and frequency.

For the sake of simplicity, we assume neither recovery from infection nor any virus-induced mortality. We characterize VVIC agents by their transmission efficiency $\beta$ (probability of virus transmission upon a sufficiently close contact between a susceptible and an infected individual), sterilizing power $\sigma$ (probability that an infected individual of the target sex becomes sterile) and sexspecificity (targeted sex).

As the model rules are stochastic (see below), we carry out 50 simulation replicates for each parameter set and model variant and evaluate average control efficiency. We define this quantity as $1-N_{c} / N_{p}$, where $N_{p}\left(N_{c}\right)$ is the host population size averaged across 50 time steps preceding the end of the pre-control (control) phase and then across the 50 replicates. Seemingly negative control efficiencies might occur for inefficient viruses (very small $\beta$ and/or $\sigma$ ) due to stochastic fluctuations around the population equilibrium, and we reset them to zero.

In order to compare the models, we define $90 \%$ control efficiency as our control goal, and the corresponding contour line in the $\beta-\sigma$ parameter space (referred to as CE90 further on) becomes the main currency on which we base our comparisons. VVIC agents with characteristics above this contour line reduce the host population to $10 \%$ or less of its pre-control size, which we regard as a reasonably good though somewhat arbitrary control measure (not necessarily appropriate for a particular application). We scan the $\beta-\sigma$ parameter space with a resolution of 0.05 in $\beta$ and 0.01 in $\sigma$.

\subsection{Non-sexual model}

Within each reproductive cycle of the pre-control phase, each individual gives birth to $b_{a}$ offspring (in the context of our non-sexual model, all individuals are female and males enter the model only implicitly). We assume that negative density dependence affects offspring survival, and let only a fraction $1-N / K$ of the offspring survive to adulthood, where $N$ is the current adult population size and $K$ is the carrying capacity. Each adult individual dies with probability $d$ at the end of the reproductive cycle.

Within each reproductive cycle of the control phase, infection is first transmitted between individuals via $\Lambda_{a}(N)$ encounters in which the two interacting individuals are taken at random. If only one of them bears the virus, the other becomes infected with probability $\beta$. This is followed by reproduction. In non-sexual models, all individuals by definition reproduce and are thus equally affected by infection - whereas susceptible individuals give birth to $b_{a}$ offspring, infected individuals produce $b_{a}$ offspring with probability $P$ and no offspring with probability $1-P$. This probability depends on the sex-specificity of the virus. Given that all modelled individuals are females, we have $P=1-\sigma$ for the female-specific virus. 
The impact of the male-specific virus has to be modelled differently; if sterilization operates by destroying spermatozoids, it prevents fertilization and leaves the ovules of a female available for another, fertile male. Following Barlow (1994), we thus set $P=1-[\sigma I /(S+I)]^{h}$, where $I$ and $S$ are the numbers of infected and susceptible individuals in the population, respectively. In a sexually reproducing population, this is the probability that any given female mates with at least one fertile male in a population composed of $S$ susceptible and $I$ infected males. Each of these males is assumed to mate with $h$ randomly chosen females within one reproductive cycle; $h$ thus represents the average number of matings per female. Density-dependent (DD) offspring survival and adult mortality are the same as in the pre-control phase.

\subsection{Two-sex model}

In the pre-control phase, each male is assumed to mate with $h$ randomly chosen females within each reproductive cycle. Each female that mates with at least one male gives birth to $b_{s}$ offspring, each becoming male or female with probability 0.5 . To maintain a correspondence with the non-sexual model, where all individuals give birth, we set $b_{s}=2 b_{a}$. Adult mortality and density dependence in offspring survival are analogous to the non-sexual model.

Within each reproductive cycle of the control phase, mating season is preceded by a period of reproductive quiescence. Infection is first transmitted between random pairs of individuals through $\Lambda_{s}(N)$ non-mating encounters. If only one of them bears the virus, the other becomes infected with probability $\beta$. During the mating season, each male mates $h$ times. As we expect VVIC efficiency to depend on the degree of promiscuity, we run simulations for several values of $h(1,2,5$, and 10 ; results for $h=2$ and 10 are shown only in electronic supplementary material). Note that $h=1$ does not imply strict monogamy with more or less firm pair bonds. Although each male mates with only one female and the population has 1:1 sex ratio, some females may mate repeatedly while others may stay unmated due to the random mate choice.

Upon mating, infection is also transmitted with probability $\beta$. Following Thrall et al. (2000), we assume the latent period of the infection to extend beyond the mating season, and do not consider secondary transmissions within this period. As a consequence, females that contract the female-specific virus in the mating season are immediately impacted by it, while males that contract the malespecific virus in that season can inflict any 'harm' not until the next reproductive cycle. Any male infected by the malespecific virus has probability $1-\sigma$ to successfully fertilize a female upon mating, and one successful mating is assumed sufficient for the female to produce $b_{s}$ offspring. In the case of the female-specific virus, mated females produce $b_{s}$ offspring if susceptible, and $b_{s}$ or no offspring with probability $1-\sigma$ and $\sigma$, respectively, if infected. Negative density dependence in offspring survival, offspring sex determination, and adult mortality operate as in the precontrol phase.

\subsection{Virus transmission and encounter functions $\Lambda_{a}(N)$ and $\Lambda_{s}(N)$}

Virus transmission is traditionally considered as frequency-dependent (FD) or density-dependent (DD) (Begon et al., 2002), i.e. susceptible individuals become infected at a rate proportional to the actual frequency or density of infected animals, respectively. We discuss the DD transmission in more detail in Appendix A; for it, the number of encounters (all encounters in the non-sexual model and non-mating encounters in the two-sex model) per individual increases linearly with population size and $\Lambda_{x}(N)=\lambda_{x} N^{2}$, where $x$ stands for $a$ or $s$. In what follows, unless explicitly specified otherwise, we focus on the FD transmission, i.e. we assume that the per-individual number of encounters in the population is density-independent and $\Lambda_{x}(N)=\lambda_{x} N$.

We proceed by assuming that $\lambda_{s}$ is either preset or estimated from data. Non-sexual models cannot distinguish between non-mating and mating encounters that may exist in sexually reproducing populations. To estimate $\lambda_{a}$, we thus use the most straightforward approach: we lump both non-mating and mating encounters together and set $\lambda_{a}$ such that the total number of encounters is the same as in the two-sex model. In a sexually reproducing population with $N$ individuals and 1:1 sex ratio, one reproductive cycle involves $\lambda_{s} N$ non-mating and $h N / 2$ mating encounters. We thus take $\lambda_{a}=\lambda_{s}+h / 2$ as an adequate choice for the FD transmission. For the DD transmission, we set $\lambda_{a}=\lambda_{s}+h /$ $(K \sqrt{ } 2)$ (see Appendix A).

To give $\lambda_{s}$ a specific value, we define the maximum number $E$ of non-mating encounters in a reproductive cycle as the number of non-mating encounters when the uncontrolled population occupies the whole habitat $(N=K)$. Values of $E$ and $K$ define $\lambda_{s}$ through $\Lambda_{s}(K)=E$, i.e. $\lambda_{s}=E / K$ for $\mathrm{FD}$ encounters and $\lambda_{s}=E / K^{2}$ for DD encounters. We investigate four different values of $E(100$, $1000,10,000$ and 100,000), which give $0.1,1,10$ and 100 FD non-mating encounters per individual and reproductive cycle at $K=1000$, respectively.

\section{Results}

Our results show that the four model variants (nonsexual vs. two-sex model variants and male- vs. femalespecific virus, all for the FD transmission) can lead to drastically different quantitative predictions. Nevertheless, they share common qualitative properties as regards the shape and position of the CE90 contour line (Figs. 1 and 2).

Firstly, only relatively high sterilizing power $\sigma$ can reduce the established pest population to less than $10 \%$ of its pre-control size while requirements on transmission efficiency $\beta$ are usually much less restrictive. Obviously, the 


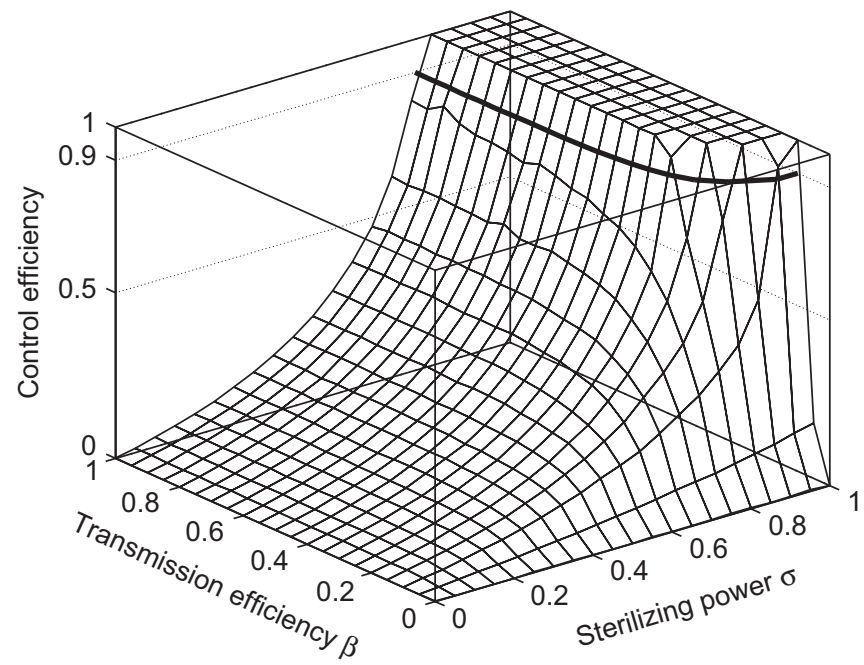

Fig. 1. Generic example of the dependence of VVIC efficiency on virus sterilizing power $\sigma$ and transmission efficiency $\beta$. The results shown are for the two-sex model with the female-specific virus and FD non-mating encounters. Parameters: $E=1000, b_{s}=2, d=0.2, h=5, K=1000$. The thick solid line highlights $90 \%$ control efficiency.

maximum control success is achieved for the maximum sterilizing power $(\sigma=1)$ and the maximum transmission efficiency $(\beta=1)$, and it may even mean complete eradication (the complete eradication contour line runs more or less parallel and close to the CE90 contour; Fig. 1). Secondly, control success is much more sensitive to changes in sterilizing power than in transmission efficiency (unless, in some cases, the latter is very low), and saturates with increasing $\beta$ (Fig. 1). Thirdly, control success always increases with the maximum number of non-mating encounters, $E$, as these help spread the virus without affecting the mating season. Finally, the impact of increased male mating activity (larger $h$ ) is not straightforward, as it helps virus proliferation but, on the other hand, has a positive feedback on reproduction. The latter effect is absent in the non-sexual model with the female-specific virus and the control success thus increases, but it prevails in the other three situations and leads to decreased control efficiency (Fig. 2A).

\subsection{Inadequacy of non-sexual models}

The discrepancies between the results of non-sexual and two-sex model variants apparently stem from two factors. Firstly, the two-sex model explicitly models the sexual reproduction. It thereby includes randomness in mating encounters and some females may not mate. This leads to lower mean reproductive success of females, which improves control efficiency relative to the non-sexual model, in which all individuals 'succeed in mating'. The difference is the largest for low $h$ when the possibility for females to stay unmated is considerable, and diminishes for large enough $h$ when virtually all females become fertilized. By producing a different pattern of mating encounters, this explicit consideration of sexual reproduction also modifies the pattern of transmission events and disease spread. Secondly, the two-sex model does not allow for secondary transmissions during the mating period, which lowers control efficiency relative to the non-sexual model, as it hinders more efficient virus dissemination. Overall, we observe the largest discrepancy between the non-sexual and two-sex models for $h=1$ and very few encounters outside the mating period (when $E$ and thus $\lambda_{s}$ is low; Fig. 2A).

On the other hand, predictions of the non-sexual and two-sex models virtually coincide (irrespective of the virus sex-specificity) when neither of these two factors plays a role. Such a situation occurs when individuals have a high number of mating partners ( $h$ is large) and the virus is markedly transmitted during the non-mating period ( $E$ is large). This effectively means that all females mate and all population members become infected. For the same reason, control efficiency becomes almost independent of transmission efficiency for large $E$ (the CE90 contour line is horizontal and bends up sharply only for very low values of $\beta)$. Indeed, individuals with extensive contacts will acquire the virus even if it has very poor transmission efficiency (Fig. 2A).

\subsection{Effects of sex-specificity}

Our results further show that female-specific viruses can almost always control the host population more effectively than male-specific ones. The use of otherwise identical male- and female-specific viruses leads to the same control success only for $h=1$ in the non-sexual model. In this case the total mean number of offspring in a given year is the same $\left(b_{a}[S+(1-\sigma) I]\right)$ whatever the sex-specificity of the virus. The difference between female- and male-specific viruses is especially pronounced in the two-sex model with few non-mating encounters (small $E$ ), where male-specific viruses can fall short of the $90 \%$ control target even if they have the maximum sterilizing power and the maximum transmission efficiency (Fig. 2A). Even when non-mating encounters are numerous (large $E$ ), male-specific viruses require more sterilizing power than female-specific ones to meet the same control target. Finally, the difference gets larger with increasing $h$. While the CE90 contour line due to the female-specific virus approaches a limiting line, that corresponding to the male-specific virus eventually vanishes as there is an increasing chance for females to meet at least one fertile male so that their successful reproduction becomes warranted (Fig. E1 in electronic supplementary material).

\subsection{Effects of demographic parameters and DD encounters}

Additional simulations showed that control efficiency in populations with different carrying capacities $K$ remains essentially the same as long as the number of non-mating encounters per individual when the population is at the 
A

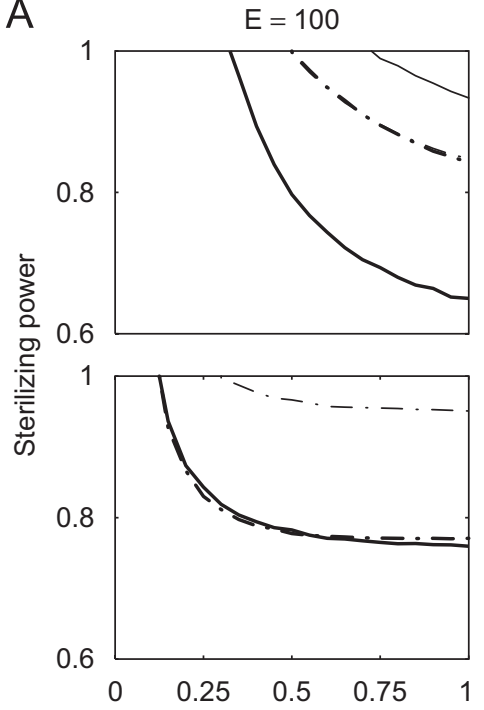

B

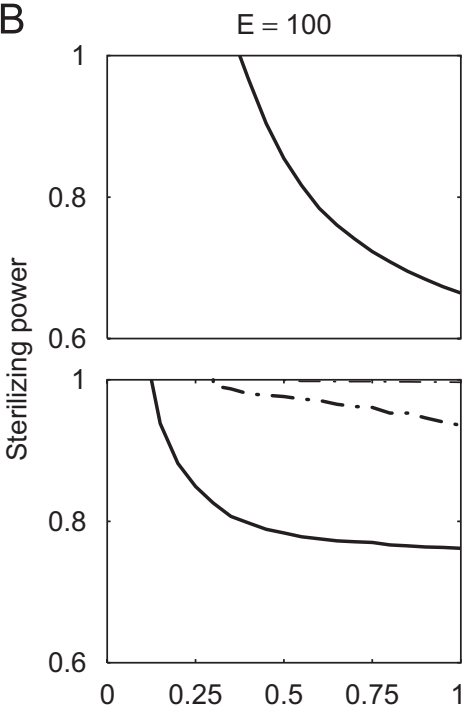

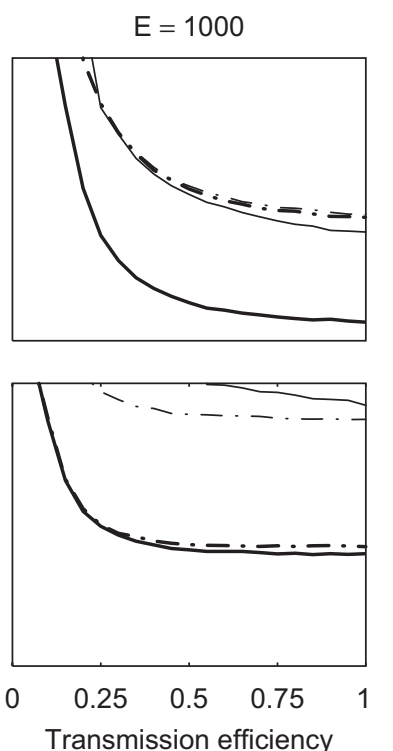
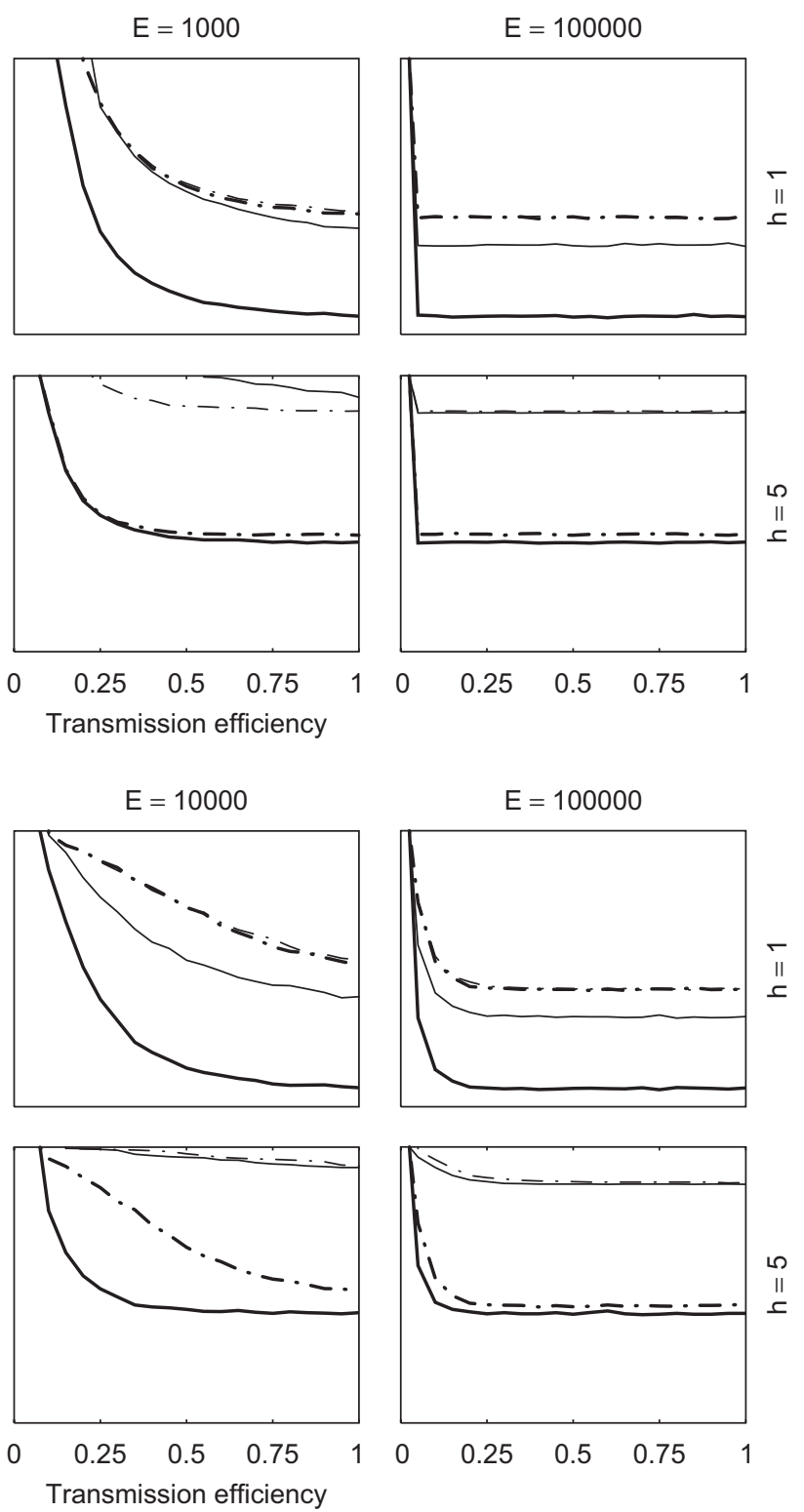

Fig. 2. Control efficiency (illustrated by CE90 contour lines in the $\beta-\sigma$ parameter space, above which the host population is reduced to $10 \%$ or less of its pre-control size) as a function of the maximum number of non-mating encounters $E$ and the degree of promiscuity $h$. (A) Frequency-dependent (FD) nonmating encounters. (B) Density-dependent (DD) non-mating encounters. Lines: thick—female-specific virus, thin — male-specific virus; solid-two-sex model, dash-dot - non-sexual model. Missing lines indicate that the corresponding model did not reach $90 \%$ control efficiency; for $h=1$ and the nonsexual model the lines for male- and female-specific viruses coincide (see the main text for the explanation). Parameters: $b_{s}=2, d=0.2, K=1000$. Note the truncated $y$-axis. Additional results are included in electronic supplementary material (Fig. E1).

carrying capacity remains constant (Fig. E2). Control efficiency generally improves with decreasing birth rate or increasing death rate (results not shown).

The results for DD non-mating encounters are qualitatively similar to those for FD ones. However, due to the mismatch between the FD mating encounters and DD nonmating encounters (see Appendix A), the discrepancy between the non-sexual and two-sex models is generally stronger than in the case of the (FD mating encounters and) FD non-mating encounters (Fig. 2B). In particular, predictions of both model variants do not coincide until much higher values of $h$ and/or $E$.

\section{Discussion}

\subsection{Non-sexual models are generally not appropriate for predicting VVIC efficiency}

Our study highlights that non-sexual models generally fail to match predictions of their two-sex counterparts on the efficiency of VVIC in mammal pest control. Most of the differences arise from the two different infection pathways - apart from strictly STD, viruses may spread through mating as well as non-mating encounters. The two pathways are clearly distinguished only in the two-sex 
model. Two major processes taking place during the mating period underlie the differences: compared to the non-sexual model, the two-sex model accounts for variability in mating success of individual females and prohibits secondary virus transmission during the mating period. Non-sexual models cannot properly disentangle these pathways and hence can misjudge the VVIC efficiency, especially if non-mating encounters are less frequent and the degree of promiscuity is low. The inadequacy of the non-sexual model is even more pronounced if the number of non-mating encounters per individual increases with the population size (i.e. if it can be described by a DD term).

We could have tried to correct more precisely for the differences related to the two transmission pathways in the non-sexual model by introducing some extra correction factor to $\lambda_{a}$. We have not taken that avenue for two reasons: firstly, it is likely that the correction factor would differ at least for male- and female-specific viruses (as well as for FD and DD non-mating encounters), if it exists at all, hampering a more systematic approach. Secondly, and more importantly, such a correction factor de facto attempts to take the two-sex model structure into account, which we believe is fundamentally equivalent to constructing a full two-sex model, yet more laborious and providing no clear mechanistic insight.

As a result of the model design, females exhibit a greater variance in mating success than males. Since variability in reproductive success is smaller in females than in males of some species (Clutton-Brock, 1988, 1989), we investigated whether changing our modelling assumptions would modify our conclusions. For a high non-mating encounters rate, the correlation between an individual's mating success and its probability of becoming infected is weak. Hence, decreasing the female variance in mating success should slightly reduce the discrepancy between the results of the non-sexual and two-sex models; increasing the male variance should have no impact at all. On the other hand, these modifications could enlarge the gap between the results of the two models for a low rate of non-mating encounters since an individual's mating success and its probability of becoming infected are likely to be positively correlated. Thus this does not qualitatively change our conclusions.

The predictions of the non-sexual and two-sex models coincide only if the animals are highly promiscuous (having high $h$ ) and, in addition, non-mating encounters dominate over mating ones such that virtually all individuals become infected. However, the minimum 'critical' values of these quantities ( $h$ and the proportion of non-mating encounters among all encounters) at which the predictions coincide cannot be given by a simple rule of thumb, as they depend on the sex-specificity and transmission pattern of the virus. The discrepancy between the non-sexual and two-sex model and potential derivation of the correction factor could become even more complex if more features specific to mating are considered. We thus conclude that nonsexual models are less appropriate for studying the impact of VVIC on promiscuous pest populations.
STDs correspond to $E=0$ in our modelling framework (non-mating encounters do not transmit the virus). In that case, DD encounters in the non-sexual model grossly underestimate the control success of the female-specific virus as given by the two-sex model (Fig. E3 in Appendix B). However, since transmission of STDs is generally FD (May and Anderson, 1987; McCallum et al., 2001; Thrall and Antonovics, 1997), the non-sexual model we considered seems to be an appropriate tool to investigate impacts of female-specific viruses responsible for STDs, provided the degree of promiscuity is sufficiently high (Fig. E3).

In this paper we focused on the comparison of predictions of a two-sex model and its non-sexual counterpart. We showed that the predictions can be fundamentally different, and this conclusion will most probably hold for other existing (non-sexual) models as well. Any direct comparison of our two-sex model with published nonsexual models would thus provide little useful insights.

\subsection{What does a successful virus look like?}

Our results show that male- and female-specific viruses affect the pest population differently: targeting females is consistently more effective than targeting males. That is because the lost reproductive contribution of sterile males, unlike that of females, can be covered by fertile males without any loss of offspring at the population level (e.g. Tuyttens and Macdonald, 1998). The asymmetry in the reproductive roles of sexes is thus reflected in the asymmetrical control efficiency. Barlow (1994) predicted a higher efficiency of a female-specific, sexually transmissible virus in possum populations, and a review of experiments by Bomford (1990) concluded that male sterilization showed little promise and was inferior to control oriented towards females. Our study extends these conclusions by showing that, at least for promiscuous mating systems, female-specific viruses are superior also when transmitted via non-mating encounters.

Despite that apparent superiority, however, male-specific viruses should not be a priori rejected if they happen to be easier and cheaper to produce and are able to reach higher sterilizing powers. For example, Holland et al. (1997) reported a possibility of engineering a myxoma virus coding one sperm antigen in order to control European rabbits $(O$. cuniculus) in Australia. As we show in this study, this possibility is certainly worth considering at least at low levels of promiscuity.

We also observed a persistent asymmetry in the effectiveness of sterilizing power and transmission efficiency. In particular, for male-specific viruses satisfactory results critically depended on high sterilizing power. This is a key result for potential applications: virus selection is less constrained by transmission efficiencies. Virus engineering efforts should rather aim to maximize its sterilizing power. This route is probably easier to follow than attempts to genetically manipulate a virus to ensure higher transmission efficiency: Hood et al. (2000) pointed out that in the 
rabbit-myxoma system, an increase in the inherent transmissibility of the virus is not likely, while Barlow (2000) reported an immunocontraceptive protein that can reduce fertility by up to $75 \%$ when injected to possums. The relatively wide window of transmission efficiencies leading to successful control allows for a wide spectrum of viruses to be tested as potential VVIC agents.

It has been claimed that controlling populations on a mainland is much more difficult than on islands (Courchamp et al., 2003). We have shown that VVIC efficiency roughly depends on the number of non-mating encounters per individual at the population carrying capacity. As a consequence, the same virus will more efficiently impact denser populations, which are more likely to occur on islands without potential enemies and competitors.

Along with the virus properties, control efficiency can also be affected by the magnitude and frequency of virus re-introductions into the pest population. Our simulations suggest that while re-introductions are important for the virus persistence, the outcome is little sensitive to their magnitude and frequency. This contrasts sharply with Courchamp and Cornell (2000), who modelled regular reinoculations by spatially distributed infected baits and found that increasing baiting rate (encompassing both magnitude and frequency of re-inoculation) leads to increased control efficiency, especially for FD virus transmissions. Differences between the two modelling approaches currently prevent us from providing any clear explanations for these contrasting results.

\subsection{What makes an ideal target species?}

Our models clearly show that an ideal target species should have plentiful non-mating interactions (to favour virus spread) and relatively few mating interactions (to prohibit successful re-mating) between individuals. Ideal target species thus should interact strongly (e.g. be social, very mobile or highly gregarious) and be less promiscuous. On the other hand, successful control of species with relatively few non-mating encounters requires high sterilizing power or, when the transmission efficiency is low, high levels of promiscuity.

Species currently considered as potential targets of VVIC include rabbits, invasive rodents (e.g. rats, mice and gray squirrels), marsupials (e.g. brushtail possums) and introduced carnivores (e.g. red foxes and domestic cats). These species belong to different trophic levels, have different life histories and mating systems, and are attacked by viruses that differ in such characteristics as virulence, stage specificity, transmission pattern and possibility of host recovery and permanent immunity. Therefore, different mammal species may respond differently to attempts on fertility control (Barlow et al., 1997; Caughley et al., 1992). Further theoretical studies should therefore explore a wider range of host types attacked by a wider range of VVIC agents in order to assess generality of our results. In addition, reasonably detailed, species-specific models should be developed to achieve highly precise and reliable predictions (McLeod and Twigg, 2006).

To keep model comparisons simple, we excluded two possible outcomes of infection: recovery and mortality. Recovery would undoubtedly reduce control efficiency since it would directly reduce the sterilization effect. Even if individuals remained sterile once recovered, recovery would indirectly reduce virus spread by reducing the proportion of infectious individuals in the population. Recovery potential can perhaps be reduced through genetic engineering of the virus, and we therefore suggest attempting this if possible.

The impact of mortality due to infection is less straightforward since its direct, short-term benefits (removal of individuals from the population and increased control efficiency, as in Barlow (1994)) might be outweighed by diminishing the opportunities for virus spread in the long run (as in Hood et al., 2000). However, the models in Barlow (1994), Hood et al. (2000) and our paper are all different; the issue of net mortality effects thus deserves more detailed future work. For example, a sterilizing virus could actually decrease natural mortality of the host by decreasing the amount of energy individuals invest into reproduction. This might be disadvantageous in the short-term but beneficial on longer timescales since it should favour the virus spread.

\subsection{Final remarks}

This study focused on relatively short-term, populationdynamic consequences of releasing VVIC agents. Our results support current efforts in VVIC research and suggest that VVIC could be a very efficient technique for non-lethal control of unwanted mammal populations. However, we cannot stress enough the risks inherent in releases of genetically modified viruses with no current possibility to remove or even control them (Barlow, 2000; Courchamp and Cornell, 2000; Simberloff and Stiling, 1996). Therefore, in addition to genetic and population studies, the need to explore long-term and/or communitywide impacts of VVIC cannot be overemphasized (Angulo and Cooke, 2002).

\section{Acknowledgments}

We acknowledge funding by the Institut Français pour la Biodiversité (no. 209/01; AD + FC), Grant Agency of the Academy of Sciences of the Czech Republic (IAA100070601; LB + DSB) and Institute of Entomology, Biology Centre ASCR (Z50070508; LB+DSB). DSB is currently supported by EU Research Training Network FishACE (contract no. MRTN-CT-2004-005578). We thank Charles Godfray and an anonymous reviewer for helpful comments and Samantha O'Loughlin for help with the English. 


\section{Appendix A. Density-dependent non-mating encounters}

Because of a mismatch between FD mating encounters and DD non-mating encounters with $\Lambda_{x}(N)=\lambda_{x} N^{2}(x=a$ or $s$ ), any correspondence between the non-sexual and two-sex models cannot be based simply on equating the total number of encounters per individual per reproductive cycle in both model variants. Nevertheless, we again look for $\lambda_{a}$ such that $\Lambda_{a}(N)$ is the 'best' approximation of $\Lambda_{s}(N)+h N / 2$ over a range of population sizes. In analogy with regression methods from statistics, we considered the curve $\Lambda_{s}(N) / N+h / 2$ as a 'data set' for the number of per individual encounters, and fitted $\Lambda_{a}(N) / N$ to it such that the quantity $\int_{0}^{K}\left|\Lambda_{s}(N) / N+h / 2-\Lambda_{a}(N) / N\right| \mathrm{d} N$ is minimized. The integration covers the range of attainable population sizes. Simple calculation shows that the integral attains its minimum for $\lambda_{a}=\lambda_{s}+C h / K$ with $C=\frac{1}{\sqrt{ } 2}$. The minimization criterion involving the total number of encounters (rather than the number of per individual encounters) yields only a slightly different value of $C$. Due to the mismatch, the results for DD encounters are qualitatively similar to FD encounters, but differ quantitatively.

\section{Appendix B. Supplementary data}

Supplementary data associated with this article can be found in the online version at doi:10.1016/j.jtbi. 2007.09.037.

\section{References}

Angulo, E., Cooke, B., 2002. First synthesize new viruses then regulate their release? The case of the wild rabbit. Mol. Ecol. 11, 2703-2709.

Arthur, A.D., Pech, R.P., Singleton, G.R., 2007. Cross-strain protection reduces effectiveness of virally vectored fertility control: results from individual-based multistrain models. J. Appl. Ecol., doi:10.1111/ j.1365-2664.2007.01334.x.

Atkinson, I.A.E., 2001. Introduced mammals and models for restoration. Biol. Conserv. 99, 81-96.

Barlow, N.D., 1994. Predicting the effect of a novel vertebrate biocontrol agent: a model for viral-vectored immunocontraception. J. Appl. Ecol. 31, 454-462.

Barlow, N.D., 1997. Modelling immunocontraception in disseminating systems. Reprod. Fertil. Dev. 9, 51-60.

Barlow, N.D. (Ed.), 2000. The ecological challenge of immunocontraception: editor's introduction, J. Appl. Ecol. 37, 897-902.

Barlow, N.D., Kean, J.M., Briggs, C.J., 1997. Modelling the relative efficacy of culling and sterilising for controlling populations. Wildlife Res. 24, 129-141.

Begon, M., Bennett, M., Bowers, R.G., French, N.P., Hazel, S.M., Turner, J., 2002. A clarification of transmission terms in host-microparasite models: numbers, densities and areas. Epidemiol. Infect. 129, $147-153$.

Bomford, M., 1990. A role for fertility control in wildlife management. Bureau of Rural Resources Bulletin Series, Bulletin no.7, Australian Government Publishing Service, Canberra.
Boukal, D.S., Berec, L., 2002. Single-species models of the Allee effect: extinction boundaries, sex ratios and mate encounters. J. Theor. Biol. 218, 375-394.

Bradley, M.P., Hinds, L.A., Bird, P.H., 1997. A bait-delivered immunocontraceptive vaccine for the European red fox (Vulpes vulpes) by the year 2002? Reprod. Fertil. Dev. 9, 111-116.

Caughley, G., Pech, R.P., Grice, D., 1992. Effects of fertility control on a population's productivity. Wildlife Res. 19, 623-627.

Clutton-Brock, T.H., 1988. Reproductive Success. The University of Chicago Press, Chicago.

Clutton-Brock, T.H., 1989. Mammalian mating systems. Proc. R. Soc. London B 236, 339-372.

Courchamp, F., Cornell, S.J., 2000. Virus-vectored immunocontraception to control feral cats on islands: a mathematical model. J. Appl. Ecol. 37, 903-913.

Courchamp, F., Chapuis, J.-L., Pascal, M., 2003. Mammal invaders on islands: impact, control and control impact. Biol. Rev. 78, 347-383.

Cowan, P.E., 1996. Possum biocontrol: prospects for fertility regulation. Reprod. Fertil. Dev. 8, 655-660.

Ebenhard, T., 1988. Introduced birds and mammals and their ecological effects. Swedish Wildlife Res. 13, 1-107.

Holland, M.K., Jackson, R.J., 1994. Virus-vectored immunocontraception for control of wild rabbits - identification of target antigens and construction of recombinant viruses. Reprod. Fertil. Dev. 6, 631-642.

Holland, M.K., Andrews, J., Clarke, H., Walton, C., Hinds, L.A., 1997. Selection of antigens for use in a virus-vectored immunocontraceptive vaccine: PH-20 as a case study. Reprod. Fertil. Dev. 9, $117-124$.

Hood, G.M., Chesson, P., Pech, R.P., 2000. Biological control using sterilizing viruses: host suppression and competition between viruses in non-spatial models. J. Appl. Ecol. 37, 914-925.

Legendre, S., Clobert, J., Møller, A.P., Sorci, G., 1999. Demographic stochasticity and social mating system in the process of extinction of small populations: the case of passerines introduced to New Zealand. Am. Nat. 153, 449-463.

Lever, C., 1994. Naturalized Animals: The Ecology of Successfully Introduced Species. Poyser Natural History, London.

Lindström, J., Kokko, H., 1998. Sexual reproduction and population dynamics: the role of polygyny and demographic sex differences. Proc. R. Soc. London B 265, 483-488.

May, R.M., Anderson, R.M., 1987. Transmission dynamics of HIV infection. Nature 326, 137-142.

McCallum, H., Barlow, N., Hone, J., 2001. How should pathogen transmission be modelled? Trends Ecol. Evol. 16, 295-300.

McLeod, S.R., Twigg, L.E., 2006. Predicting the efficacy of virallyvectored immunocontraception for managing rabbits. N. Z. J. Ecol. $30,103-120$.

O'Keefe, K.J., Antonovics, J., 2002. Playing by different rules: the evolution of virulence in sterilizing pathogens. Am. Nat. 159, 597-605.

Pech, R., Hood, G.M., McIlroy, J., Saunders, G., 1997. Can foxes be controlled by reducing their fertility? Reprod. Fertil. Dev. 9, 41-50.

Rankin, D.J., Kokko, H., 2007. Do males matter? The role of males in population dynamics. Oikos 116, 335-348.

Rodger, J.C., 1997. Likely targets for immunocontraception in marsupials. Reprod. Fertil. Dev. 9, 131-136.

Sarre, S.D., Aiken, N., Clout, M.N., Ji, W., Robins, J., Lambert, D.M., 2000. Molecular ecology and biological control: the mating system of a marsupial pest. Mol. Ecol. 9, 723-733.

Simberloff, D., Stiling, P., 1996. How risky is biological control? Ecology 77, 1965-1974.

Smith, G., Walmsley, A., Polkinghorne, I., 1997. Plant-derived immunocontraceptive vaccines. Reprod. Fertil. Dev. 9, 85-89.

Thrall, P.H., Antonovics, J., 1997. Polymorphism in sexual versus non-sexual disease transmission. Proc. R. Soc. London B 264, $581-587$. 
Thrall, P.H., Antonovics, J., Wilson, W.G., 1998. Allocation to sexual versus nonsexual disease transmission. Am. Nat. 151, 29-45.

Thrall, P.H., Antonovics, J., Dobson, A.P., 2000. Sexually transmitted diseases in polygynous mating systems: prevalence and impact on reproductive success. Proc. R. Soc. London B 267, 1555-1563.

Tuyttens, F.A.M., Macdonald, D.W., 1998. Fertility control: an option for non-lethal control of wild carnivores? Anim. Welfare 7, 339-364.
Tyndale-Biscoe, C.H., 1994. Virus-vectored immunocontraception of feral mammals. Reprod. Fertil. Dev. 6, 281-287.

Verdier, Y., Chaffaux, S., Artois, M., Boue, F., 1999. Characterization of sperm antigens for use as a target for immunocontraception in the red fox (Vulpes vulpes). Hum. Reprod. 14, R112.

Vitousek, P.M., Dantonio, C.M., Loope, L.L., Rejmanek, M., Westbrooks, R., 1997. Introduced species: a significant component of human-caused global change. N. Z. J. Ecol. 21, 1-16. 